\title{
Raisonnement clinique: des conversations au sein de cultures épistémiques ${ }^{\star}$
}

\section{Clinical reasoning: conversations in epistemic culture}

\author{
Joy $\operatorname{HIGGS}^{1, *}$ \\ Charles Sturt University, Sydney Olympic Park, Sydney, NSW, Australia \\ Manuscrit soumis le 5 mars 2018 ; accepté pour publication le 7 mars 2018
}

\begin{abstract}
Résumé - Problématique : Le raisonnement clinique est un mode de pensée et de prise de décisions en situation professionnelle, lié au contexte et servant à guider les actions professionnelles. Exégèse: Cette contribution décrit le raisonnement clinique comme un processus et une activité intégrés dans la pratique et les cultures épistémiques. Il se concrétise grâce aux conversations entre les personnes prenant part à la prise de décisions. Un raisonnement clinique avancé implique d'aller au-delà des actions liées à la prise de décisions cliniques, à la recherche de «l'aisance épistémique». Cette dernière permet d'élever le raisonnement clinique à un niveau de compétence intégrée, réflexive et interactive se réalisant par l'entremise de conversations à propos de la prise de décisions cliniques.
\end{abstract}

Mots clés : raisonnement clinique, culture épistémique

\begin{abstract}
Context: Clinical reasoning is a context-dependent way of thinking and decision-making in professional practice to guide practice actions. Analysis: Clinical reasoning is embedded in practice and epistemic cultures and is enacted through conversations with the decision-making participants. Advanced clinical reasoning involves moving beyond the acts of clinical decision-making in the pursuit of epistemic fluency to enhance clinical reasoning in action as an embodied, reflexive and interactive capability that is realised through clinical decision-making conversations.
\end{abstract}

Keywords: clinical reasoning, epistemic culture

\section{Introduction}

Le raisonnement clinique est un mode de pensée et de prise de décisions en situation professionnelle, lié au contexte et servant à guider les actions professionnelles. Il implique la construction de récits permettant d'interpréter les multiples facteurs et particularités relatifs à l'activité de raisonnement. Le raisonnement clinique est mis en jeu dans le cadre d'une famille de situations que le

\footnotetext{
^ Ce texte a été élaboré à partir d'une conférence plénière prononcée dans le cadre de la troisième Conférence internationale de Montréal sur le raisonnement clinique, qui s'est tenue à Montréal du 25 au 28 octobre 2016. La traduction en français a été effectuée par Caroline Faucher, en collaboration avec MarieClaude Audétat, Annie Carrier, Joseph-Omer Dyer, Stuart Lubarsky et Meredith Young, en lien avec le comité de rédaction de la revue.

*Correspondance et offprints: Joy HIGGS, Charles Sturt University, Sydney Olympic Park, Sydney, NSW, Australia. Mailto: jhhiggs@csu.edu.au.
}

praticien problématise en s'appuyant à la fois sur des cadres de référence particuliers qui lui sont propres, sur le contexte de travail, des modèles de pratique et les contextes du patient ou du client. Il exploite les dimensions essentielles des savoirs pratiques, du raisonnement et de la métacognition, et s'appuie également sur la disponibilité de telles capacités chez les autres. Au cours même du raisonnement clinique, le processus de prise de décision peut concerner les niveaux micro, macro et méta et ce, individuellement ou en collaboration. Il sollicite des métahabiletés de conversations critiques, de production de connaissances, ainsi que des modèles de pratique caractérisés par une authenticité et une réflexivité [1].

Cet article présente quatre arguments principaux qui soutiennent une telle interprétation du raisonnement clinique :

- le raisonnement clinique est une pratique culturellement enracinée ;

- une telle culture est principalement de nature épistémique ; 


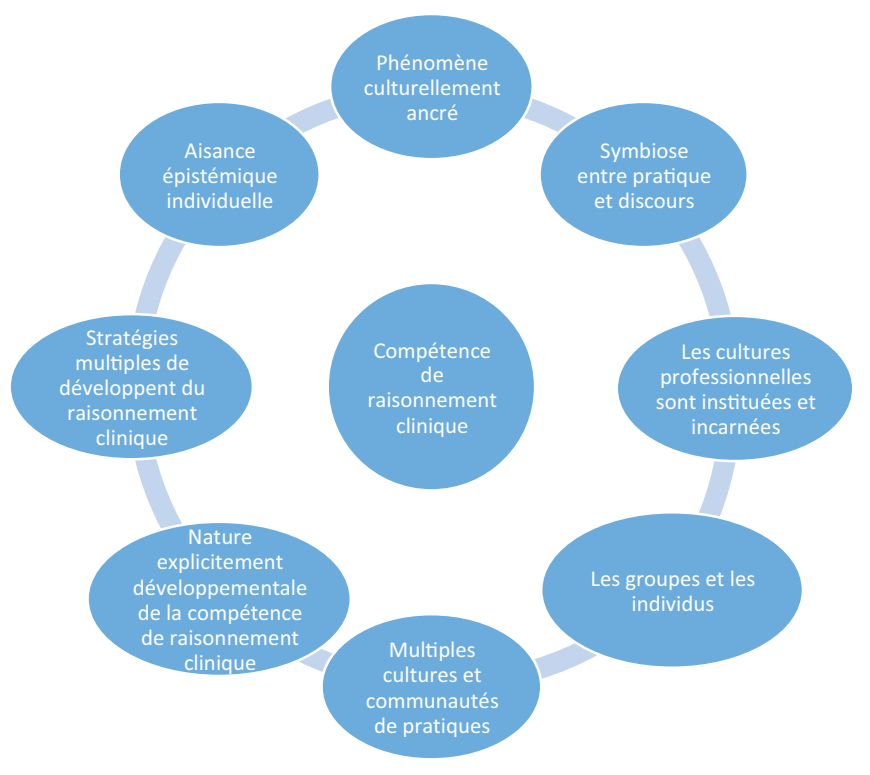

Figure 1. La compétence de raisonnement clinique.

- la mise en œuvre du raisonnement peut, idéalement et en réalité, être considérée comme étant un processus conversationnel ;

- la compétence de raisonnement clinique est liée à l'aisance épistémique.

La figure 1 représente ces idées regroupées au sein de la compétence de raisonnement clinique.

\section{Le raisonnement clinique en tant que pratique culturellement enracinée}

Le raisonnement clinique et la prise de décision sont sollicités dans divers environnements culturels : la société, les systèmes de santé (incluant les structures, les buts, les sources de financement, les attentes, les participants et les organisations), les normes de pratique institutionnelles, de même que les contextes de pratique intra- et interprofessionnels.

Il est essentiel de saisir la nature des professions et d'en définir les caractéristiques pour bien concevoir le raisonnement clinique comme étant une pratique spécifique, intégrée dans un ensemble plus large d'activités constitutives de la pratique professionnelle. La société accorde à certains emplois un statut professionnel, avec les privilèges et les responsabilités qui leur incombent. Dans le système de santé, les professions se caractérisent par des pratiques et des rôles qui leur sont propres. Les professionnels membres de chaque profession élaborent un discours spécifique, associé à leurs pratiques, et développent en collégialité une base de connaissances spécialisées, qui leur est propre, tout en recourant à des savoirs génériques issus des champs de la santé, des sciences biomédicales et des sciences sociales. Ils développent des pratiques particulières de raisonnement, ainsi que des modalités propres de connaissance en action, ou de production de savoirs à partir de la pratique ou dans le cadre d'activité de recherche et de formation. Les professions partagent les responsabilités et les attentes relatives au professionnalisme, y compris le comportement éthique et l'obligation de diligence envers leurs patients et leurs collègues.

\section{Cultures épistémiques}

Nous concevons les professions comme des communautés de pratique agissant localement, nationalement et mondialement. De plus, les communautés de pratique peuvent fonctionner selon un mode interprofessionnel au sein d'organisations. Dans les deux cas, de telles communautés de pratique démontrent leurs capacités à collaborer grâce à un partage de discours, de langage, de buts et de pratiques. Ces artéfacts - au sens où il s'agit de constructions sociales - et ces actions, les uns et les autres partagés, constituent ensemble les dimensions de la culture. On peut mettre en perspective les notions de cultures de pratique et celle de paradigmes (empiricoanalytique, interprétatif et critique, par exemple). Ainsi, dans une culture de pratique, un groupe de praticiens (qui peuvent être des chercheurs partageant un paradigme de recherche ou des praticiens/cliniciens partageant un paradigme de pratique) utilise les normes, savoirs et pratiques intégrés dans leur culture pour fonctionner ensemble de manière cohérente. Les membres d'un groupe de pratique marchent, parlent, et pensent d'une façon commune qui prend racine dans une même culture. Cette façon d'agir résulte de deux processus clés. Le premier est le processus sociohistorique de la professionnalisation, par laquelle les emplois sont devenus des professions. Le second est le processus de socialisation professionnelle, par lequel les individus deviennent des membres de leur profession. Dans le premier cas, la profession se définit ellemême et devient un groupe auto-réglementé avec des normes de pratiques établies, des critères de formation, conditions du maintien d'une appartenance. Dans le second processus, l'individu apprend et s'engage à respecter les codes de conduite et de pratique spécifiques à la discipline et à la profession. Dans les deux cas, l'élément clé est la reconnaissance et le respect de la vision du «monde» de la pratique professionnelle ainsi établie (l'ontologie de la pratique) et son lien avec la façon de déterminer et créer les savoirs au sein de ce monde de pratique (l'épistémologie de la pratique) [2].

Les cultures épistémiques émergent de ces pratiques et artéfacts communs. La notion de culture épistémique est une manière riche et profonde de comprendre les mondes de pratique et la cohabitation symbiotique dans ces mondes entre le discours sur les savoirs et la pratique instituée. Les cultures épistémiques comprennent à la fois les savoirs tels qu'ils sont mis en pratique, et les cadres disciplinaires, socioculturels et définis par l'expertise, dans lesquels les savoirs et les pratiques interagissent au bénéfice des acteurs de ces cultures.

Les cultures professionnelles sont ainsi adoptées et incarnées au sein de la profession en tant que groupe, de même que par les praticiens à titre individuel. Cette institutionnalisation implique un dialogue vivant et critique entre les savoirs, les activités et le raisonnement 
liés aux pratiques. Chacun de ces trois éléments fait partie des dimensions évolutives d'une pratique vivante dans des cadres de pratique sociale changeants.

\section{Conversations dans les cultures épistémiques}

Si l'on considère de telles cultures vivantes en relation avec les soins de santé, la culture requiert la communication et la prise de décision nécessite des conversations. Ces conversations impliquent à tout le moins la vérification des besoins, buts et attentes des patients ou clients, de même que la contribution du praticien ou du professionnel expert à la prise de décision et au choix de traitement. Souvent, la pratique nécessite aussi des conversations et une prise de décision collaborative, entre des praticiens se partageant les responsabilités relatives à la gestion du cas. Idéalement, les conversations prennent en compte et respectent les multiples cultures, les perspectives expertes et la connaissance de soi des participants (parmi lesquels les patients ou clients, et les soignants), de même que les perspectives et les prérogatives de chaque personne impliquée. De cette façon, la prise de décision clinique, riche et collaborative, est enracinée dans la culture tout en nécessitant une conversation.

\section{La compétence de raisonnement clinique et les cultures épistémiques}

Le cheminement au sein du concept de culture épistémique conduit à aborder la notion d'aisance épistémique [3]. Markauskaite et Goodyear [4] la décrivent ainsi : on peut dire des personnes flexibles et habiles quant aux différentes façons de connaître le monde qu'elles possèdent une aisance épistémique.

La maîtrise du raisonnement clinique est une compétence faisant partie intégrante d'une pratique expérimentée et experte. L'exercice général de pratiques professionnelles compétentes nécessite la maîtrise du raisonnement clinique. Une telle compétence correspond à un savoir-agir en situation, ancré dans la pratique, qui peut être à la fois documenté et argumenté [5]. Allant au-delà de la simple capacité technique, elle englobe une agentivité, une ingéniosité et une confiance en ses actions, ces dernières incluant la prise de décision, l'identification des problèmes et la résolution de problèmes. La compétence implique la capacité de bien agir, à la fois dans des contextes connus et inconnus, et la capacité de résoudre des problèmes cliniques simples ou complexes. La compétence se manifeste dans les actions, les relations et le travail collaboratif efficace.
Elle se manifeste également dans la confiance en ses aptitudes à gérer les circonstances non familières et à apprendre à partir de ces expériences.

Le développement et la maîtrise de la compétence de raisonnement clinique exigent des praticiens qu'ils s'impliquent dans une compréhension en profondeur du raisonnement en tant que pratique complexe, mais aussi qu'ils reconnaissent la nature intrinsèquement contextualisée du raisonnement clinique. Ils doivent de plus valoriser différentes approches du raisonnement adaptées au niveau de compétence pour la tâche du praticien concerné [6], maîtriser efficacement le langage et la verbalisation du raisonnement auprès de divers partenaires, et employer des stratégies d'apprentissages mettant en pratique chacune de ces habiletés et conceptions.

\section{Conclusion}

Le raisonnement clinique est un ensemble de processus réflexifs profondément contextualisés dans la discipline des praticiens, leurs façons de connaître et d'apprendre, leurs propres modèles de pratique et leurs milieux de travail. Durant leur transition entre le niveau du novice, qui apprend le raisonnement clinique comme étant un processus systématique, conscient et fondé sur la gestion des risques, et le niveau du véritable expert professionnel dont la pratique est très avisée et judicieuse, les praticiens doivent parvenir à concevoir que le raisonnement clinique est la dimension la plus essentielle et intégrative de la pratique professionnelle.

\section{Références}

1. Higgs J. The complexity of clinical reasoning: exploring the dimensions of clinical reasoning expertise as a situated, lived phenomenon. Seminar presentation at the Faculty of Health Sciences: The University of Sydney, Australia, 2006.

2. Higgs J, Richardson B, Abrandt Dahlgren FM. Developing practice knowledge for health professionals. ButterworthHeinemann: Oxford, 2004.

3. Goodyear P, Zenios M. Discussion, collaborative knowledge work and epistemic fluency. Br J Educ Stud 2007;55:351-68.

4. Markauskaite L, Goodyear P. Epistemic fluency and professional education: innovation, knowledgeable action and actionable knowledge. Springer: Dordrecht (The Netherlands), 2017.

5. Stephenson J. The concept of capability and its importance in higher education. In: Capability and quality in higher education, Stephenson J, Yorke M, Editors. Kogan Page: London (UK), 1998, p. 1-13.

6. Higgs J. The teacher in self-directed learning: manager or comanager? In: Learner managed learning: practice, theory and policy, Graves N, Editor. World Education Fellowship: London (UK), 1993, p. 122-31.

Citation de l'article : Higgs J., Raisonnement clinique : des conversations au sein de cultures épistémiques. Pédagogie Médicale $2017: 18 ; 51-53$ 